\title{
Tunable Multifunctional Topological Insulators in Ternary Heusler Compounds
}

Stanislav Chadov ${ }^{1}$, Xiaoliang Qi ${ }^{2,4}$, Jürgen Kübler ${ }^{3}$, Gerhard H. Fecher ${ }^{1}$, Claudia Felser ${ }^{1}$ \& Shou Cheng Zhang ${ }^{4}$

${ }^{1}$ Institut für Anorganische Chemie und Analytische Chemie, Johannes Gutenberg - Universtität, 55099 Mainz, Germany

${ }^{2}$ Microsoft Research, Station Q, Elings Hall, University of California, Santa Barbara, CA 93106, USA

${ }^{3}$ Institut für Festkörperphysik, Technische Universität Darmstadt, 64289 Darmstadt, Germany

${ }^{4}$ Department of Physics, McCullough Building, Stanford University, Stanford, California 943054045, USA

Recently the Quantum Spin Hall effect (QSH) was theoretically predicted and experimentally realized in a quantum wells based on binary semiconductor HgTe ${ }^{1+3}$ QSH state and topological insulators are the new states of quantum matter interesting both for fundamental condensed matter physics and material science. ${ }^{1-11}$ Many of Heusler compounds with $\mathrm{C1}_{b}$ structure are ternary semiconductors which are structurally and electronically related to the binary semiconductors. The diversity of Heusler materials opens wide possibilities for tuning the band gap and setting the desired band inversion by choosing compounds with appropriate hybridization strength (by lattice parameter) and the magnitude of spin-orbit coupling (by the atomic charge). Based on the first-principle calculations we demonstrate that around 
fifty Heusler compounds show the band inversion similar to HgTe. The topological state in these zero-gap semiconductors can be created by applying strain or by designing an appropriate quantum well structure, similar to the case of HgTe. Many of these ternary zero-gap semiconductors (LnAuPb, LnPdBi, LnPtSb and LnPtBi) contain the rare earth element $\mathrm{Ln}$ which can realize additional properties ranging from superconductivity (e. g. LaPtBi ${ }^{[12}$ ) to magnetism (e. g. GdPtBi $\left.{ }^{13}\right)$ and heavy-fermion behavior (e. g. YbPtBi $\left.{ }^{14}\right)$. These properties can open new research directions in realizing the quantized anomalous Hall effect and topological superconductors.

According to their electronic structure all bulk materials are divided into metals, which have a finite electron density at the Fermi energy and insulators, which show the band gap. Recently a new class of the so-called topological states has emerged - the Quantum Spin Hall (QSH) state in two dimensions and its generalization in three dimensions. The corresponding materials, called topo-

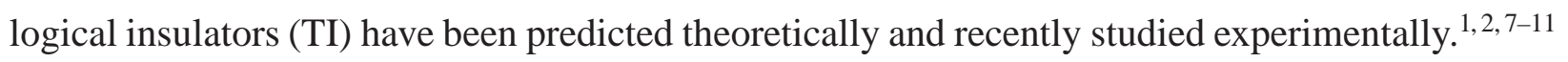
The TI is a new state of quantum matter with a full insulating gap in the bulk, but with topologically protected gapless surface or edge states on the boundary. Research on TI's has attracted considerable attention due to both fundamental interest to a new state of matter as well as its possible application in new spintronic devices. ${ }^{4}$ All the TI's discovered so far were either alloys $\left(\mathrm{Bi}_{1-x} \mathrm{Sb}_{x}\left[\frac{7 \sqrt[8]{8}}{)}\right)\right.$ or binary compounds $\left(\mathrm{HgTe},{ }^{1 / 2} \mathrm{Bi}_{2} \mathrm{Se}_{3}, \mathrm{Sb}_{2} \mathrm{Te}_{3}\right.$ and $\left.\mathrm{Bi}_{2} \mathrm{Te}_{3}{ }^{[-11}\right)$ This letter reports about tunable multifunctional TI's within the class of ternary semiconducting Heusler compounds. The great diversity of these materials (more than 200 semiconductors among 500 Heusler compounds) opens wide possibilities for tuning their bandstructure comparing to binary compounds 
and enables an effective search of the optimal TI material for applications.

In particular, several compounds, $\mathrm{YPtSb}, \mathrm{YPdBi}$ and $\mathrm{ScAuPb}$ are found at their experimental lattice constants $\frac{15}{15}$ close to the border between the trivial and topological insulator, with all relevant bands degenerated at the $\Gamma$ point (see Fig. 3(a)). Such a material can be easily transformed from a trivial to a topological insulator and vice versa by a small variation of the lattice constant (by applying pressure or growing the film on the appropriate substrate). Many TI candidate compounds ( $\mathrm{LnAuPb}, \mathrm{LnPdBi}, \mathrm{LnPtSb}$ and $\mathrm{LnPtBi}$ ) containing the rare earth elements Ln with strongly correlated $f$-electrons can exhibit various conventional orders, such as magnetism, ${ }^{[13}$ superconductivity or heavy fermion behavior ${ }^{14}$ Such conventional orders in TI's enable the realization of many novel topological effects and exotic particles, such as the image monopole effect, $\frac{16}{\text { axion }}{ }^{17}$ and Majorana fermion! ${ }^{18}$ Thus the ternary TI's are multifunctional and can be exploited to design new devices. Combinations of the ternary trivial/topological insulators (such as $\mathrm{ScPtSb} / \mathrm{ScPtBi}$ similar to $\mathrm{CdTe} / \mathrm{HgTe}$ ) can be used as quantum well devices for the QSH.

Ternary Heusler compounds of $\mathrm{X}_{2} \mathrm{YZ}$ or $\mathrm{XYZ}$ composition (with $\mathrm{X}, \mathrm{Y}$ - the transition or rare earth metals and $\mathrm{Z}$ - the main group element) form the class of materials which are well-suited for various spintronic applications. ${ }^{19}$ The semiconducting nature of these compounds arise due to the strong tendency towards covalent bonding. From basic structural and bonding considerations the $\mathrm{X}_{2} \mathrm{YZ}$ Heusler compounds (L2 $2_{1}$ structure) with 18 or 24 valence electrons ${ }^{20}$ and XYZ Heuslers $\left(\mathrm{Cl}_{b}\right)$ with 18 valence electrons ${ }^{21-23}$ are expected to exhibit a gap at the Fermi energy. In the following we will focus on the XYZ Heuslers (also called half-Heuslers). The relation to 
the classical semiconductors is easy to understand for such materials as e. g., LiZnAs. More surprisingly, $\mathrm{LaPtBi}$ (sometimes also named $\mathrm{LaBiPt}$ ) is a semiconductor formed by three metallic elements! Typically the XYZ half-Heuslers can be viewed as comprised from $\mathrm{X}^{n+}$ ion "stuffing" the zincblende $\mathrm{YZ}^{n-}$ sublattice (here $\mathrm{Li}^{+}$stuffed in $[\mathrm{ZnAs}]^{-}$or $\mathrm{La}^{3+}$ stuffed in $[\mathrm{PtBi}]^{3-}$ sublattice) where the number of valence electrons associated with $\mathrm{YZ}^{n-}$ is equal to $18\left(d^{10}+s^{2}+p^{6}\right)$. 18-electron compounds are closed shell species, non-magnetic and semiconducting.

Figure 1 illustrates a comparison of the zincblende and the Heusler structures. The additional stuffed rare earth atoms are shown as orange spheres. Similar to the binary semiconductors, the band gap can be tuned by the electronegativity difference of the constituents and the lattice constant ${ }^{23}$ in a wide range from about $4 \mathrm{eV}(\mathrm{LiMgN})$ down to zero (LaPtBi). The semiconducting $\mathrm{Fe}_{2} \mathrm{VAl}^{24}$ and $\mathrm{ZrNiSn}^{25}$ Heusler compounds in addition demonstrate the excellent thermoelectric properties, just like the known $\mathrm{TI}^{\prime} \mathrm{Bi}_{2} \mathrm{Te}_{3}$ and $\mathrm{Bi}_{2} \mathrm{Se}_{3} \cdot{ }^{2627}$ To understand ternary compounds as TI's, we compare them to the binary compounds studied in the literature. For example, CdTe is a trivial semiconductor, whereas HgTe is topological system.11/37 Such drastic change in properties occurs due to interplay of the spin-orbit coupling (produced by the heavy $\mathrm{Hg}$ and $\mathrm{Te}$ atoms) and the degree of hybridization (controlled by the lattice constant). The same considerations apply to all ternary semiconducting Heusler compounds. Further we verify this based on first-principle bandstructure calculations by using the fully-relativistic version ${ }^{28}$ of the standard LMTO (Linearized Muffin-Tin Orbitals) approach. The exchange-correlation part of the effective potential was treated using Vosko-Wilk-Nusair parameterization ${ }^{29}$ of the Local Density Approximation (LDA). The following example (Figure 2) compares calculated bandstructures of CdTe and HgTe with ScPtSb and 
ScPtBi. For direct comparison with model calculations ${ }^{1}$ we mark the relevant bands carrying $\Gamma_{6}$ representation as blue and $\Gamma_{8}$ as red. As it follows, the bandstructures of these ternary compounds reveal clear fingerprints: both $\mathrm{CdTe}$ and $\mathrm{ScPtSb}$ exhibit a direct gap at the $\Gamma$ point between the conduction (blue) and the valence (red) bands of $\Gamma_{6}$ and $\Gamma_{8}$ symmetries, respectively. On the other hand, the bandstructures of $\mathrm{HgTe}$ and ScPtBi exhibit the same band inversion: $\Gamma_{6}$ (blue) is now situated below $\Gamma_{8}$ (red) which remains at the Fermi energy. This is the necessary condition for the TI state, since it changes the parity of the wave function compared to $\mathrm{CdTe}$ or $\mathrm{ScPtSb}$ (for details see the work of Dai and coworkers ${ }^{3}$ ).

To illustrate the impressive number of new topological compounds and the possibility to tune the $\Gamma_{6}-\Gamma_{8}$ band ordering in this class of materials, we have performed similar calculations for all relevant Heuslers containing Sc, Y, La, Lu and Th. The energy difference between $\Gamma_{6}$ and $\Gamma_{8}$ bands as a function of lattice constant is shown on Figure 3(a). Each subgroup (e.g. Ln = Sc, Y, La, Lu) is marked by a certain color. Compounds containing $f$-electrons, such as $\mathrm{Pr} \mathrm{Tb}$, etc. (except of $\mathrm{Ce}$ ) are not presented here, since their partially filled $f$-electron shells with strong correlations require special treatment that goes beyond standard LSDA approach. The compounds with $E_{\Gamma_{6}}-E_{\Gamma_{8}}>0$ are trivial insulators, whereas those with $E_{\Gamma_{6}}-E_{\Gamma_{8}}<0$ are the TI candidates. The latter group consists of the zero-gap semiconductors with double-degenerated $\Gamma_{8}$ point at the Fermi energy. It follows that all existing Heuslers with zero band gap at the Fermi energy (around 50 compounds by including the rare earth based) under certain conditions will demonstrate the same type of band inversion as HgTe. Indeed, the increase of the lattice constant reduces the hybridization and closes the non-zero band gap. Combined with sufficiently strong spin-orbit coupling it leads to a 
pronounced $\Gamma_{6}-\Gamma_{8}$ band inversion, which is the key to realize the TI state. Figure 3(b) demonstrates the $\Gamma_{6}-\Gamma_{8}$ difference as a function of the average spin-orbit coupling expressed by the sum of the nuclear charges of the elements in the unit cell, i.e. $\mathrm{Z}(\mathrm{X})+\mathrm{Z}(\mathrm{Y})+\mathrm{Z}(\mathrm{Z})$. The latter appears to be a suitable order parameter which sorts the materials almost along the straight line. As it follows from Figure 3, the combinations of $\mathrm{Pt}$ with $\mathrm{Bi}$ in $\mathrm{LnPtBi}$ or $\mathrm{Au}$ with $\mathrm{Pb}$ in $\mathrm{LnAuPb}$ series always lead to the inverted band structure. There is an additional advantage of Heusler materials which also follows from Figure 3(a): due to the large amount of compounds with different gap values it is easy to construct a quantum well comprised of the trivial and topological parts with well-matching lattice constants, similar to the $\mathrm{HgTe} / \mathrm{CdTe}$ quantum well. The appropriate pairs can be chosen from the candidates situated in the middle area of Figure 3(a) along the same vertical line, since the transition from trivial to a topological behavior as a function of lattice constant appears to be rather smooth in average. The relevant combinations, for example, are $\mathrm{ScPdBi} / \mathrm{ScPtBi}, \mathrm{YPdSb} / \mathrm{YPtSb}$ and $\mathrm{LuAuSn} / \mathrm{LuPtBi}$.

Although the analogy with $\mathrm{HgTe}$ is quite clear and convincing, we would like to demonstrate the topological nontrivial nature of the zero gap Heuslers in a more rigorous way. On this purpose, we study the topological phase transition between trivial and inverted phases in $\mathrm{YPdBi}$, one the materials which sits right at the border between the trivial and topological insulator states (see Fig. 3). Figure. 4(a) demonstrates the corresponding transition in details for YPdBi induced by a small variation of the lattice constant. The insets show the corresponding bandstructures. At the equilibrium lattice constant a linear dispersing Dirac cone is formed by the $\Gamma_{8}$ light-hole and $\Gamma_{6}$ bands, degenerate with the $\Gamma_{8}$ quadratic heavy-hole band. Small variation of the lattice constant (by 
about $\pm 0.3 \%$ ) substantially influences the band structure near the Fermi energy: the compression leads to a trivial state with the band gap $E_{\Gamma_{6}}-E_{\Gamma_{8}} \approx 0.07 \mathrm{eV}$, whereas the expansion leads to a zero-gap inverted state with a difference $E_{\Gamma_{6}}-E_{\Gamma_{8}} \approx-0.07 \mathrm{eV}$. Analogous effect is achieved by scaling the spin-orbit coupling magnitude $\lambda$ at the critical lattice constant (Fig. 4(b)). The suppression of spin-orbit coupling $(\lambda<1)$ drives the system into a trivial state while the increase $(\lambda>1)$ leads to a topological insulator.

Furthermore, for HgTe it has been proposed that a uniaxial strain can lift the degeneracy between light-hole and heavy-hole subbands of $\Gamma_{8}$, which drives this zero-gap semiconductor into a real TI phase ${ }^{3 / 7}$ Similar effects can be studied by our ab-initio calculations in Heusler compounds. Figure 5 shows the YPdBi bandstructure calculated for a small tetragonal strain applied along [001]-direction reducing the $c / a$ ratio by $3 \%$. One can see that both normal (Fig. 5(a)) and inverted (Fig. 5(c)) systems are gapped. The gapless Dirac cone at the "critical" lattice constant (Fig. 5(b)) remains robust, while the quadratic heavy-hole band is pushed away from Fermi level. It should be noticed that the dispersion at the critical point is linear along all directions in the momentum space around $\Gamma$, although the linear region is very small along the directions perpendicular to the strain direction. Consequently, such a transition driven by the change of lattice constant corresponds to the sign change of the mass term in $3 \mathrm{D}$ Dirac equation, which is exactly the theory of the topological phase transition between trivial and topological insulators (this is a similar approach as that used for 2D HgTe quantum well ${ }^{1}$ ). Thus we conclude that the inverted half-Heuslers are topologically nontrivial. 
The proposed materials can be tuned from a trivial to a topological insulator mainly in two different ways: (i) by variation of the lattice constant (applying pressure or growing the material on appropriate substrate); (ii) by substitution of elements (varying their electronegativities or the strength of spin-orbit coupling). (iii) The devices allow for the additional options to manipulate the electronic structure, such as to switch the borderline compound from trivial to topological by applying a gate voltage or by constructing the quantum well structures.

After the initial discovery of the QSH systems, the current research is now focused on the proximity effect between the TI's and other forms of ordered state, such as the magnetism and the superconductivity ${ }^{[4}$ However, the presently known TI's only become magnetic or superconducting when doped with extrinsic elements such as $\mathrm{Mn}, \mathrm{Fe}$ and $\mathrm{Cu}$. In contrast, the TI's based on Heusler compounds naturally include the $f$-shell rare earth elements intrinsically, together forming a stoichiometric system. Besides the chemical functions (passing of the three electrons to the zincblende lattice and determining the lattice size) the additional open $f$-shell element renders multifunctionality by providing the coexistence of conventional ordering with TI state, that is necessary for the realization of novel topological effects and the new extended applications. Here we list several examples of such multifunctional materials. (i) Bulk magnetism found in $\mathrm{LnPtBi}(\mathrm{Ln}=\mathrm{Nd}$, $\mathrm{Sm} \mathrm{Gd}, \mathrm{Tb}, \mathrm{Dy})^{\sqrt{13}}$ may realize the dynamical axion, ${ }^{\sqrt[17]{7}}$ which is the spin-wave excitation with a topological coupling with electromagnetic field. Such an effect provides a new design of tunable optical modulator. (ii) The heavy-fermion behavior in $\mathrm{YbPtBi} i^{14}$ may realize the recently proposed topological Kondo insulator. ${ }^{30}$ (iii) The superconductivity in the noncentrosymmetric low-carrier LaPtBi system. ${ }^{[1]}$ The absence of inversion symmetry is theoretically proposed to support topo- 
logical superconductivity ${ }^{31}$ Thus it is interesting to investigate whether the superconductivity in LaPtBi is topological.

1. Bernevig, B. A., Hughes, T. L. \& Zhang, S. C. Quantum Spin Hall Effect and Topological Phase Transition in HgTe Quantum Wells. Science 314, 1757 (2006).

2. König, M. et al. Quantum Spin Hall Insulator State in HgTe Quantum Wells. Science 318, 766 (2007).

3. Dai, X. et al. Helical edge and surface states in HgTe quantum wells and bulk insulators. Phys. Rev. B 77, 125319 (2008).

4. Qi, X.-L. \& Zhang, S.-C. The quantum spin Hall effect and topological insulators. Physics Today 63, 33 (2010).

5. Kane, C. L. \& Mele, E. J. Quantum Spin Hall Effect in Graphene. Phys. Rev. Letters 95, $226801(2005)$.

6. Bernevig, B. A. \& Zhang, S. C. Quantum Spin Hall Effect. Phys. Rev. Letters 96, 106802 (2006).

7. Fu, L. \& Kane, C. L. Topological insulators with inversion symmetry. Phys. Rev. B 76, 045302 (2007).

8. Hsieh, D. et al. A topological Dirac insulator in a quantum spin Hall phase. Nature 452, 970 (2008). 
9. Zhang, $\mathrm{H}$. et al. Topological insulators in $\mathrm{Bi}_{2} \mathrm{Se}_{3}, \mathrm{Bi}_{2} \mathrm{Te}_{3}$ and $\mathrm{Sb}_{2} \mathrm{Te}_{3}$ with a single Dirac cone on the surface. Nature Physics 5, 438 (2009).

10. Xia, Y. et al. Observation of a large-gap topological-insulator class with a single Dirac cone on the surface. Nature Physics 5, 398 (2009).

11. Chen, Y. L. et al. Experimental Realization of a Three-Dimensional Topological Insulator, $\mathrm{Bi}_{2} \mathrm{Te}_{3}$. Science 325, 178 (2009).

12. Goll, G. et al. Thermodynamic and transport properties of the noncentrosymmetric superconductor LaBiPt. Physica B 403, 1065 (2008).

13. Canfield, P. C. et al. Magnetism and heavy fermion-like behavior in the RBiPt series. J. Appl. Physics 70, 5800 (1991).

14. Fisk, Z. et al. Massive electron state in YbBiPt. Phys. Rev. Letters 67, 3310 (1991).

15. Villars, P. \& Calvert, L. D. Pearson's Handbook of Crystallographic Data for Intermetallic Phases (Amer Soc Metals, Metals Park, Ohio, 1991).

16. Qi, X.-L., Li, R., Zang, J. \& Zhang, S.-C. Inducing a Magnetic Monopole with Topological Surface States. Science Nature 323, 1184 (2009).

17. Li, R., Wang, J., Qi, X.-L. \& Zhang, S.-C. Dynamical Axion Field in Topological Magnetic Insulators. Nat. Phys. to be published (2010).

18. Fu, L. \& Kane, C. L. Superconducting Proximity Effect and Majorana Fermions at the Surface of a Topological Insulator. Phys. Rev. Letters 100, 096407 (2008). 
19. Felser, C., Fecher, G. H. \& Balke, B. Spintronics: A Challenge for Materials Science and Solid-State Chemistry. Angew. Chemie Internat. Ed. 46, 668 (2007).

20. Galanakis, I., Dederichs, P. H. \& Papanikolaou, N. Slater-Pauling behavior and origin of the half-metallicity of the full-Heusler alloys. Phys. Rev. B 66, 174429 (2002).

21. D, J., Koo, H. J. \& Whangbo, M. H. Study of the 18-electron band gap and ferromagnetism in semi-Heusler compounds by non-spin-polarized electronic band structure calculations. J. Mol. Struc. Theochem 527, 113 (2000).

22. Galanakis, I., Dederichs, P. H. \& Papanikolaou, N. Origin and properties of the gap in the half-ferromagnetic Heusler alloys. Phys. Rev. B 66, 134428 (2002).

23. Kandpal, H. C., Felser, C. \& Seshadri, R. Covalent bonding and the nature of band gaps in some half-Heusler compounds. J. Phys. D: Appl. Phys. 39, 776 (2006).

24. Kato, H., Kato, M., Nishino, Y., Mizutani, U. \& Asano, S. Effect of silicon substitution on thermoelectric properties of Heusler-type $\mathrm{Fe}_{2} \mathrm{VAl}$ alloy. J. Jpn. Inst. Metals 65, 652 (2001).

25. Sakurada, S. \& Shutoh, N. Effect of Ti substitution on the thermoelectric properties of (Zr,Hf)NiSn half-Heusler compounds. Appl. Physics Lett. 86, 2105 (2005).

26. Middendo A, L. G., Kohler H. Thermoelectric Power of n-type $\mathrm{Bi}_{2} \mathrm{Se}_{3}$ in Strong Transverse Magnetic-Fields. phys. stat. sol. (b) 57, 203 (1973).

27. Hor, Y. S. et al. p-type $\mathrm{Bi}_{2} \mathrm{Se}_{3}$ for topological insulator and low-temperature thermoelectric application. Phys. Rev. B 79, 195208 (2009). 
28. Perlov, A., Yaresko, A. \& Antonov, V. Spin-polarized Relativistic Linear Muffin-tin Orbitals Package for Electronic Structure Calculations, PY-LMTO. (unpublished).

29. Vosko, S. H., Wilk, L. \& Nusair, M. Accurate spin-dependent electron liquid correlation energies for local spin density calculations: a critical analysis. Can. J. Phys. 58, 1200 (1980).

30. Dzero, M., Sun, K., Galitski, V. \& Coleman, P. Topological Kondo Insulators. arXiv:0912.3750.

31. Qi, X. L., Hughes, T. \& Zhang, S. C. Fermi Surface Topological Invariants for Time Reversal Invariant Superconductors. arXiv:0908.3550.

Acknowledgements This work is supported by ARO, grant number W911NF-09-1-0508. Financial support by the Deutsche Forschungsgemeinschaft DFG (Research unit FOR 559, project P 07) is gratefully acknowledged.

Correspondence Correspondence and requests for materials should be addressed to Claudia Felser (felser@ unimainz.de) and Shou Cheng Zhang (sczhang@stanford.edu). 
Figure 1 Zincblende (YZ) structure (left) in comparison to the $\mathrm{C1}_{b}$ structure (right) of Heusler (XYZ) compounds. Yellow and blue spheres correspond to the main group (Z) and transition $(\mathrm{Y})$ elements, respectively. The orange spheres in $\mathrm{C}_{b}$ stand for the additional stuffing $(\mathrm{X})$ element.

Figure 2 Bandstructures of CdTe and HgTe compared to ScPtSb and ScPtBi Heuslers. Red color mark the bands with $\Gamma_{8}$ symmetry, blue - with $\Gamma_{6}$. Comparison reveals obvious similarity between binary systems and their ternary equivalents: both CdTe and ScPtSb are trivial semiconductors with $\Gamma_{6}$ situated above $\Gamma_{8}$ which sits at the Fermi energy (set to zero). Both $\mathrm{HgTe}$ and ScPtBi are topological with inverted bands order, the band with $\Gamma_{6}$ symmetry is situated below $\Gamma_{8}$.

Figure $3 E_{\Gamma_{6}}-E_{\Gamma_{8}}$ difference calculated for various Heuslers at their experimental lattice constants. HgTe and CdTe binaries are shown for comparison. Hollow squares mark the systems not reported in the literature. (a) $E_{\Gamma_{6}}-E_{\Gamma_{8}}$ difference as a function of the lattice constant. Pairs of materials with well-matching lattices for the QSH quantum wells can be easily picked up along the same vertical lines. The borderline compounds (between trivial and topological) insulators (YPtSb, $\mathrm{YPdBi}, \mathrm{ScAuPb})$ are situated closer to the zero horizontal line. (b) $E_{\Gamma_{6}}-E_{\Gamma_{8}}$ difference as a function of the average spin-orbit coupling strength represented by a sum of the nuclear charges over the unit cell.

Figure $4 E_{\Gamma_{6}}-E_{\Gamma_{8}}$ difference for $\mathrm{YPdBi}$. The insets show the bandstructures at the marked points along $\Delta$ and $\Sigma$ symmetry directions of the Brillouine zone. (a) $E_{\Gamma_{6}}-E_{\Gamma_{8}}$ 
changes by scaling the lattice constant from positive (trivial insulator) to negative (topological insulator). The borderline (critical) lattice constant (marked by dashed vertical line) corresponds to the zero gap with a Dirac cone at the Fermi energy. (b) Analogously, by scaling the spin-orbit coupling at the critical lattice constant, the system can be transformed from trivial $(\lambda<1)$ to topological insulator $(\lambda>1)$.

Figure 5 Bandstructure of YPdBi under tetragonal strain $(c / a=0.97)$. (b) At the critical lattice constant the strain shifts down the heavy-hole band with parabolic dispersion, leaving the single Dirac cone at the Fermi energy. This effect is especially pronounced along $\Lambda$ direction, parallel to the strain. We note, that along $\Delta$ (perpendicular to the strain) the bands at the Fermi energy still have a linear dispersion near the $\Gamma$ point, although on much smaller scale. Variation of the lattice constant (by $\pm 0.4 \%$ ) around the critical value leads to a trivial state by compression (a) or topological by expansion (c). 

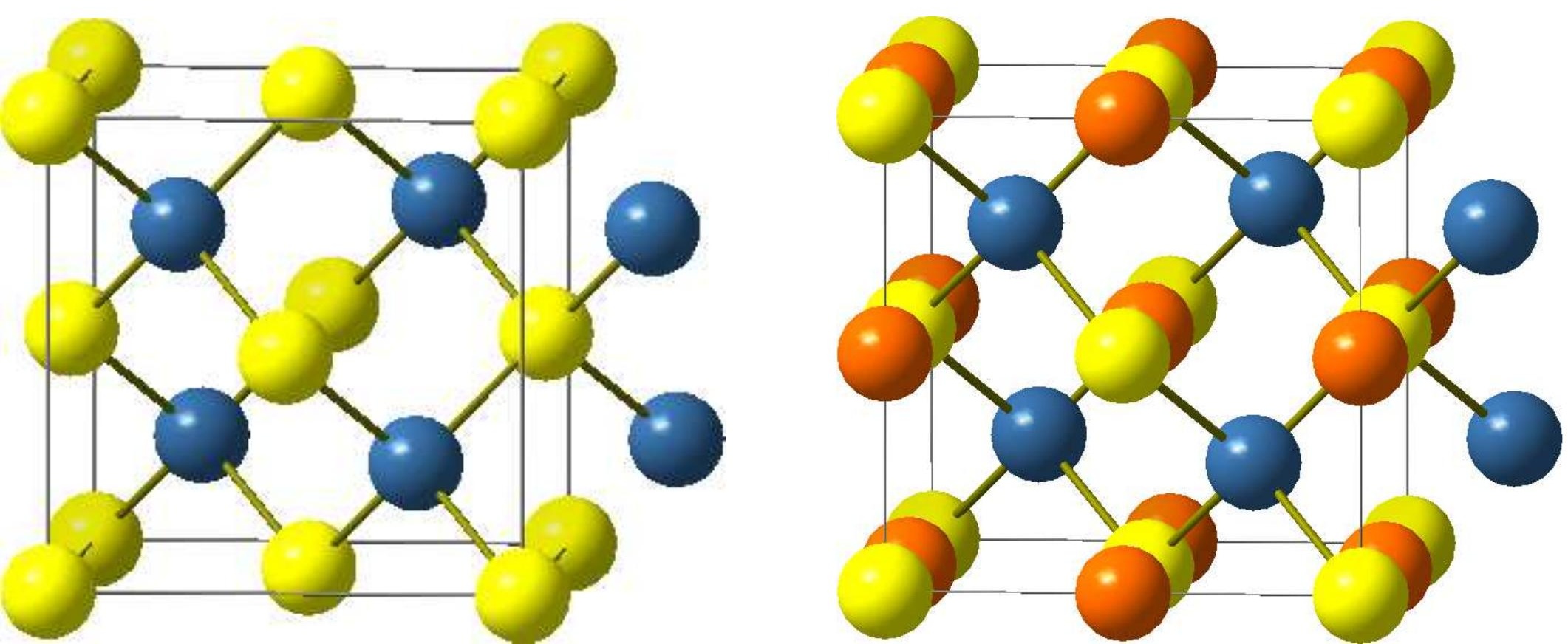


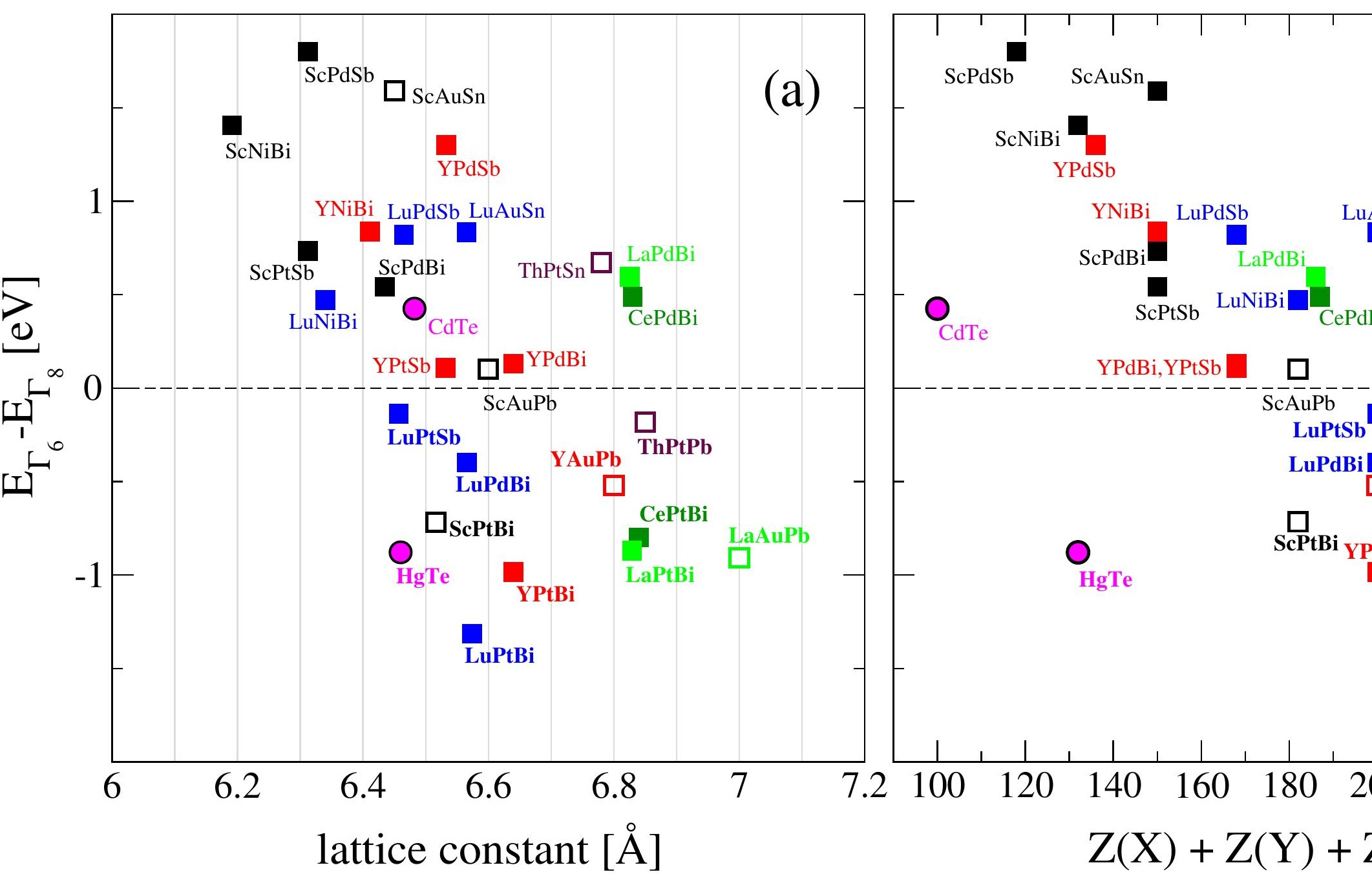


$\mathrm{E}_{\Gamma_{6}}-\mathrm{E}_{\Gamma_{8}}[\mathrm{eV}]$

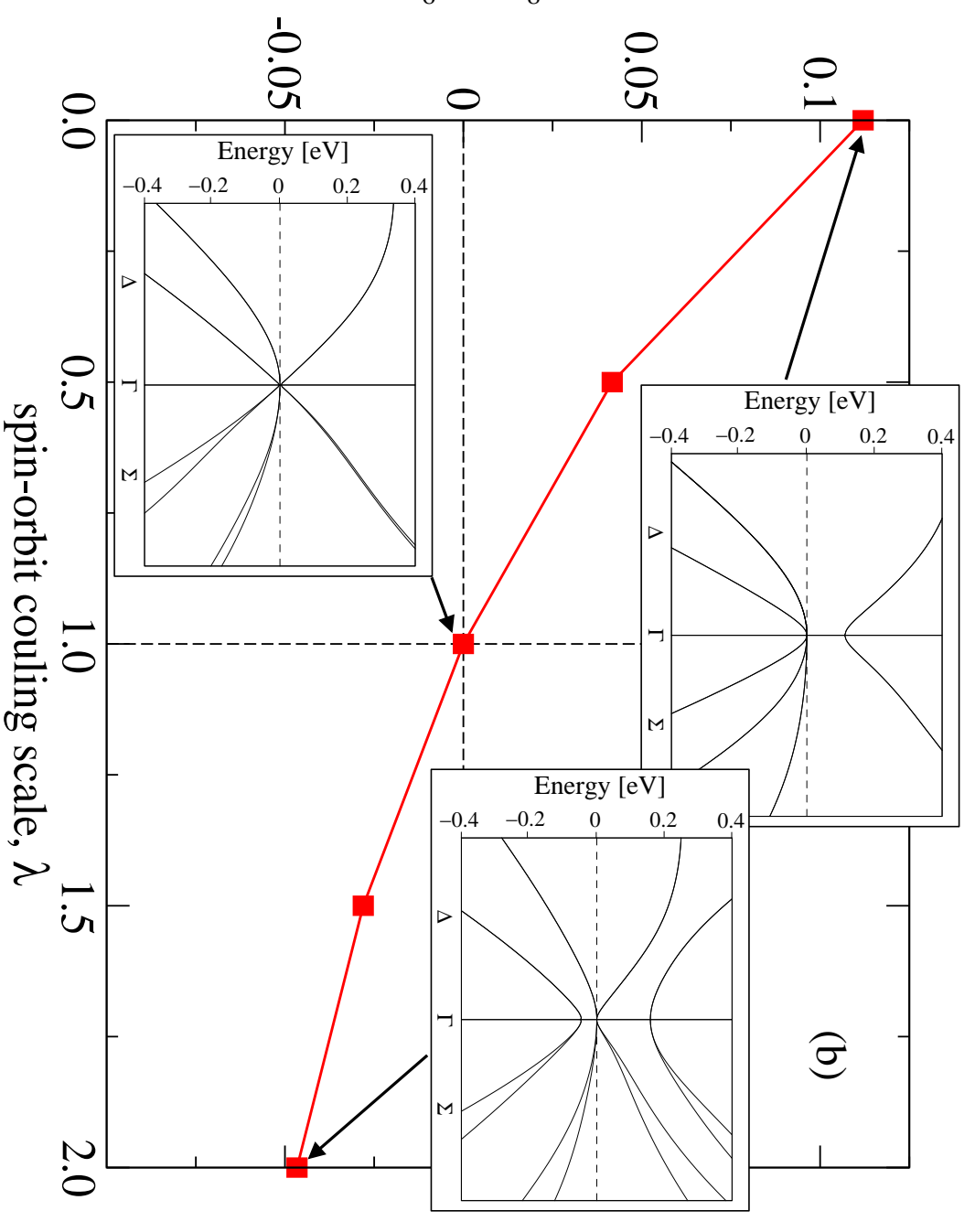

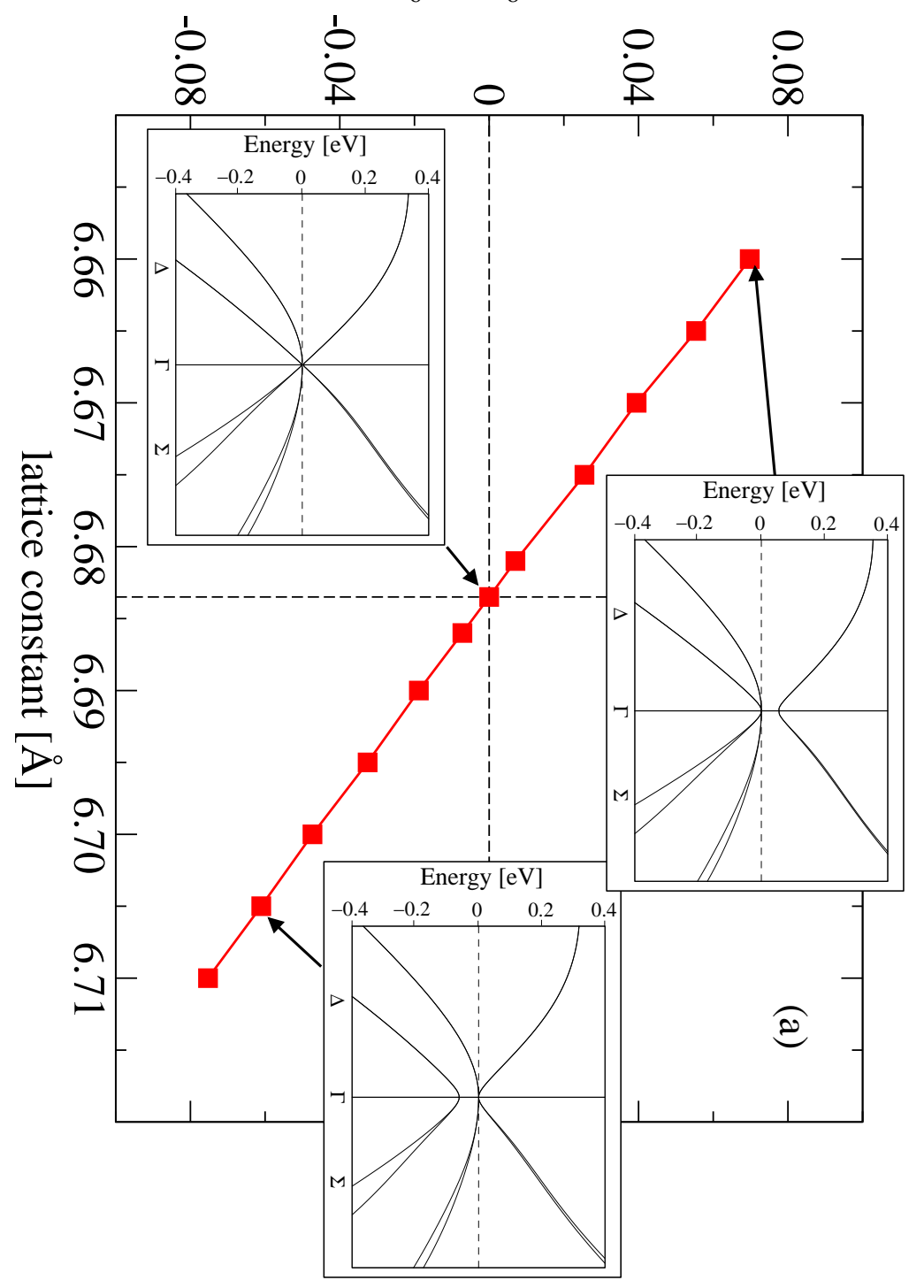



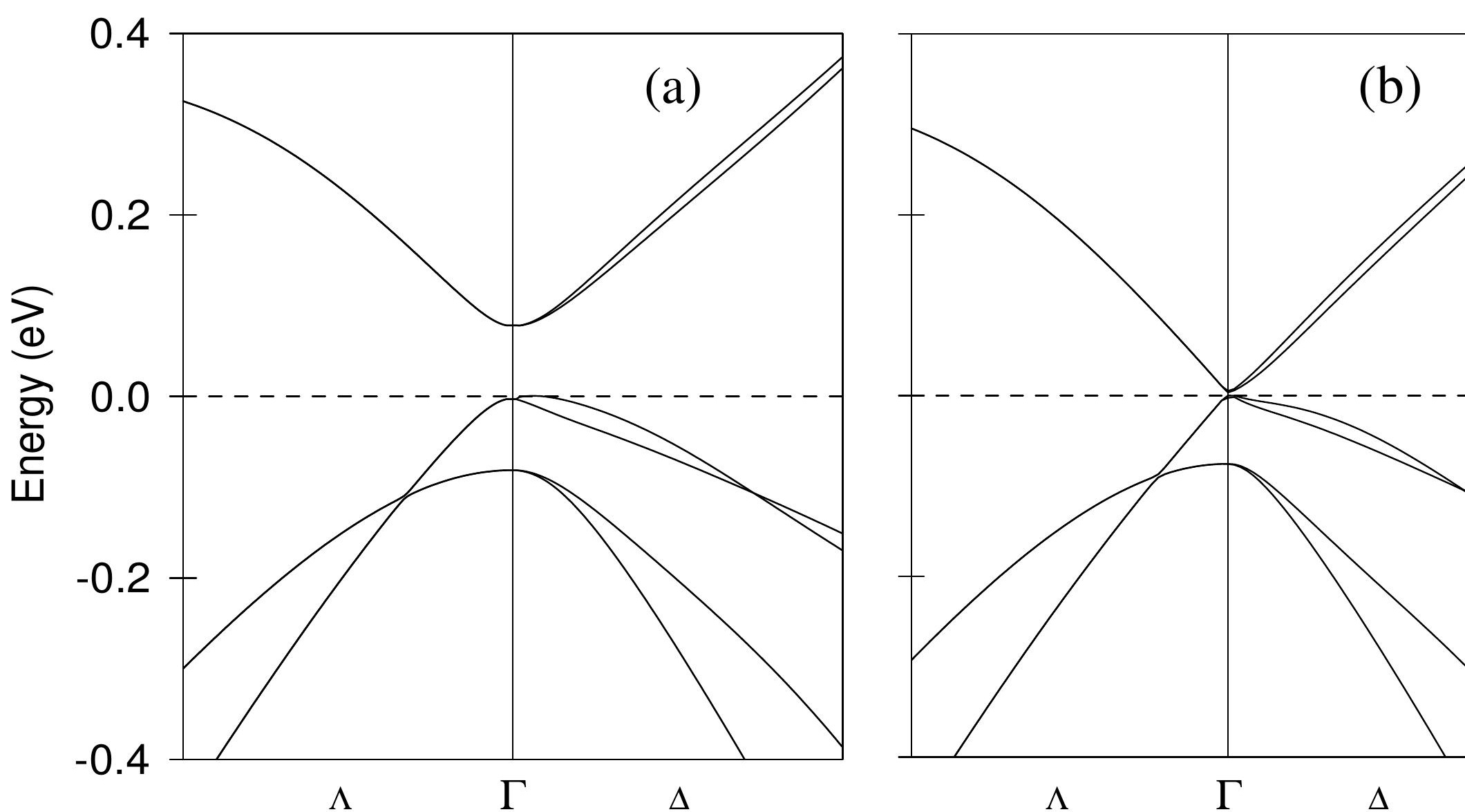